\title{
Greek Version of the Acute Cystitis Symptom Score. Part I: Linguistic Validation
}

Stamatiou K. ${ }^{1}$, Samara E. ${ }^{1}$, Alidjanov J.F. ${ }^{2}$, Naber K.G. ${ }^{3}$, Pilatz A. ${ }^{2}$, Wagenlehner F.M. ${ }^{2}$

${ }^{1}$ Tzaneio General Hospital of Piraeus, Department of Urology, Piraeus, Greece;

2Justus-Liebig University, Clinic for Urology, Pediatric Urology and Andrology, Giessen, Germany;

${ }^{3}$ Technical University of Munich, Department of Urology, Munich, Germany

\section{Running title}

The linguistic validation of the Greek ACSS

\section{Authors}

Konstantinos Stamatiou

Tzaneio General Prefecture Hospital of Piraeus

Leoforos Afentouli, Zanni ke, Pireas 185 36, Greece

e-mail: stamatiouk@gmail.com

ORCID: https://orcid.org/0000-0001-7574-3069.

Evangelia Samara

Tzaneio General Prefecture Hospital of Piraeus

Leoforos Afentouli, Zanni ke, Pireas 185 36, Greece

e-mail: gelysamara@yahoo.com

ORCID: https://orcid.org/0000-0001-9641-7342.

Jakhongir F. Alidjanov

Justus-Liebig University of Giessen,

Clinic for Urology, Pediatric Urology and Andrology

Rudolph-Buchheim str. 7, 35392 Giessen, Germany.

e-mail: Jakhongir.Alidjanov@med.uni-giessen.de; dr.alidjanov@gmail.com

ORCID: https://orcid.org/0000-0003-2531-4877.

Kurt G. Naber

Technical University of Munich, School of Medicine, Munich, Germany.

Karl-Bickleder-Straße 44C, 94315 Straubing

e-mail: kurt@nabers.de

ORCID: https://orcid.org/0000-0003-1304-5403.

Adrian Pilatz

Justus-Liebig University of Giessen,

Clinic for Urology, Pediatric Urology and Andrology

Rudolph-Buchheim str. 7, 35392 Giessen, Germany.

e-mail: Adrian.Pilatz@chiru.med.uni-giessen.de

ODCID: https://orcid.org/0000-0001-8072-1841.

Florian M. Wagenlehner

Justus-Liebig University of Giessen,

Clinic for Urology, Pediatric Urology and Andrology

Rudolph-Buchheim str. 7, 35392 Giessen, Germany.

e-mail: Florian.Wagenlehner@chiru.med.uni-giessen.de

ODCID: https://orcid.org/0000-0002-2909-0797.

\section{Corresponding author:}

Jakhongir F. Alidjanov

Rudolph-Buchheim str. 7, 35392 Giessen, Germany.

Tel.: +49 64198544551

e-mail: Jakhongir.Alidjanov@med.uni-giessen.de

dr.alidjanov@gmail.com

ORCID: https://orcid.org/0000-0003-2531-4877 


\begin{abstract}
Objective: The Acute Cystitis Symptom Score (ACSS) was developed and validated as a self-reporting questionnaire for diagnosing and monitoring acute, uncomplicated cystitis (AC) in female patients. The study aims at the translation of the ACSS into Greek from original Russian as source and American English as new master version and at its linguistic validation.
\end{abstract}

Material and Methods: Three independent professional native Greek translators, two of them experts in Russian and one in English, translated the ACSS from Russian and American English into Greek. The second group of three translators translated each of the three versions back into the original language to detect or correct any important discrepancies. These three Greek versions were then used for linguistic validation.

Results: The English to Greek translation reflected more the spoken language, the two Russian to Greek translations more the written, formal language. A total of 60 randomly selected females and 30 healthcare professionals was asked about their preferences and to comment on each of the three translations. Considering all comments the scientific committee (SC) developed the Greek consensus version. For cognitive assessment additional 30 healthcare professionals and 30 females were asked to comment on the intelligibility of all items. Considering their comments the linguistically validated Greek study version was established by the SC.

Conclusion: The linguistically validated Greek version of the ACSS can now be used for the clinical validation study.

Keywords: Greek language; translation; acute cystitis symptom score; ACSS; cystitis; urinary tract infection; female patients; diagnosis; patient-reported outcome 


\section{Introduction}

Acute cystitis (AC), an infection of the urinary bladder, is the most frequent bacterial infection in women [1]. It affects most commonly the young, sexually active women between 16 and 35 years, $10 \%$ of which get an infection every year and $40-60 \%$ at some point in their lives [2]. The classic clinical symptoms of $A C$ are dysuria, urinary frequency, urinary urgency, and suprapubic pain [3]. The urine may be cloudy and/or smelly. Also, sometimes blood is visible in urine (hemorrhagic cystitis). Frequency, duration and intensity of symptoms vary from patient to patient. In very old or very young patients symptoms may be vague or non-specific. The majority of symptoms are, however, bothersome. Recurrences are common, with up to $40 \%$ of patients getting a second infection within a year [4]. In female patients with uncomplicated cystitis, the risk of pyelonephritis after non-antibiotic therapy is in the range of $1.5-6 \%$ [5-7].

Most guidelines recommend antibiotics as first-line treatment, but symptomatic therapy also shows similar good results [5-7]. The duration of antibiotic treatment and the possible addition of other drugs such as urinary tract pain reliever often depends on the level of discomfort and persistence of symptoms. The Acute Cystitis Symptom Score (ACSS) was developed and validated as a simple and self-reporting questionnaire for diagnosing and monitoring acute uncomplicated cystitis (AC) in female patients by assessing typical and differential symptoms, quality of life and additional health conditions which may play an important role in such a clinical setting $[8,9]$. This study aims at the translation of the ACSS into Greek from original Russian as source and American English as new master version and at its linguistic validation by female patients with Greek as their first language.

\section{Materials and Methods}

The ACSS was translated from Russian into Greek according to international guidelines and recommendations [10-12]. The quality of the translation was supported by the parallel translation of the recently published American English version [13] into Greek using a cross-cultural adaptation process. Three independent native Greek translators, two of them experts in Russian and one in English, and two native Russian and one native English translator, all experts in Greek, were appointed. The first group of translators translated the ACSS from Russian and American English into the Greek language. The second group of translators translated the three versions (RG and $E G$ ) back into the original languages. Then the translators compared the original and the three back-translated versions ( $R G$ and $E G$ ) to correct any discrepancies with the original text and provided the first drafts of each questionnaire in Greek (RG and $E G$ ) after necessary corrections. These drafts were printed and distributed to randomly selected females of different educational levels who were asked whether they preferred the Russian into Greek (RG) or the English into Greek $(E G)$ version and to comment on the translated versions. After careful discussion of the preferences and comments, the scientific committee (SC) prepared a Greek consensus version, which had to undergo the face to face cognitive assessment and internal consistency evaluation. The independent Ethics Committee of the Tzaneion General Hospital of Piraeus, Piraeus, Greece, approved the study protocol (Approval no 1300/date 19-02-2020)

\section{Results}

The first 15 randomly selected females of different ages (30-89 years old) and different levels of education (from primary school to postgraduate education) were interviewed (Table 1). They were asked to comment and choose between the EG and the first RG translation. Eight of them chose the EG translation and 7 chose the RG version. No significant differences existed in the median age (50 vs 54 years) and educational level between the two groups of the responder's preferred translations, although the number of the technical university and university graduates was slightly higher among those having chosen the RG translation compared to those, who preferred the translated EG questionnaire (5/7 vs $4 / 8)$. Note that the EG translation considered more the spoken language, the $R G$ translation, however, more the written formal language. Differences between spoken and written language are more prominent in Greek and Russian compared to American English. While there was no difference in the level of education (postgraduate education) between the two translators, the age of the RG translator was significantly higher than that of the EG translator (76 vs 38 years). To rule out age-related effects and to achieve a reliable comparison, the translation from Russian into Greek was repeated by a younger translator of 37 years, not related to the previous one, and translated back into Russian by a different translator. This version of RG questionnaire still resembled mainly written Greek but was closer to the EG translation. Considering all the comments it was decided by the SC to further use the new RG translation. 
The two pre-final versions in Greek, RG and EG, were randomly applied to 30 females, including the first 15 women between 22 and 89 years (Table 1), who visited the hospital mainly as companions of patients. Their education level varied between Postgraduate education and Primary School (Table 1). Participants were invited to compare and comment on the two Greek versions and to indicate the most comprehensible version. Twenty females preferred the translated EG questionnaire, 10 preferred the translated RG questionnaire. The proportion of 2:1 did not depend on the age of the participants, but the educational level between the participants was statistically significantly different. Participants with a higher level of education (university graduates) preferred the translated RG questionnaire (6/10) to the EG questionnaire $(4 / 20)$.

Since many of the responders preferred specific translated questions from each questionnaire, as a next step 30 additional female participants (Table 2) with different education level and 30 healthcare professionals (urologists, gynaecologists, nurses, pharmacists) were asked to comment on each of the specific questions of the two Greek versions. Although all interviewees understood the questions, most of the nonhealthcare professionals' interviewees asked for any unusual or new words to be explained. All preferences and comments were discussed by the SC and finally one consensus version of the Greek ACSS was established.

The cognitive assessment was performed either face to face or by a link [14] sent by mail to Greek physicians and other healthcare professionals (pharmacists, nurses) and female subjects with history or without a history of acute cystitis (Table 3). The first 30 respondents of each group formed the study group. They were asked whether they understood all questions of the consensus version of the Greek ACSS and to comment on all questions of the questionnaire. After discussing all comments from healthcare professionals and patients, the final study version of the Greek ACSS questionnaire (Fig. 1) was established by the SC and can now be used for the clinical validation study.

\section{Discussion}

During the translation of the ACSS into Greek and its evaluation by native Greek females, we met so far unknown challenges concerning the translation of questionnaires. The difficulties are probably connected to peculiarities of the modern Greek language. In fact, in the modern era, the Greek language entered a state of diglossia: the coexistence of vernacular and archaizing written forms of the language. What came to be known as the Greek language question was a polarization between two competing varieties of Modern Greek: Dimotiki, the vernacular form of Modern Greek proper, and Katharevousa, meaning 'purified', a compromise between Dimotiki and Ancient Greek, which was developed in the early 19th century, and was used for literary and official purposes in the newly formed Greek state. In 1976, Dimotiki was declared the official language of Greece, having incorporated features of Katharevousa and giving birth to Standard Modern Greek, which is used today for all official purposes and in education [15]. Several of these incorporated features of Katharevousa are used in a formal language, which is more common in written communication, especially in public documents including medical forms. Its forms and vocabulary often 'filter down' into the vernacular, though often in a changed form high prestige, which is generally used by sophisticated persons or certain professionals e.g. lawyers [16]. Of note, formal language and informal language are associated with particular choices of grammar and vocabulary. Differences between formal language and informal language -which is more common in oral communication- are prominent in the modern Greek language. As shown in this study, a questionnaire with an old-fashioned style such as the RG version of ACSS is better acceptable by persons with tertiary education who are familiar to formal written communication. Currently, most users of American English are rather informal than formal [17]. As a result, the consensus version which was mainly resulted from the EG pre-final version was more informal, more descriptive and longer than the RG pre-final version and uses more explanatory words and sentences to the explain medical terms.

\section{Conclusions}

The process of translation and adaptation of the study instrument such as a questionnaire for a different ethnic group is a difficult task since it requires to adapt it in a culturally relevant and comprehensible form despite peculiarities of the target language. Considering these difficulties, we were able to develop a linguistically validated Greek version of the ACSS, which now can be used for the clinical validation study. 


\section{Acknowledgements}

Authors thank all the participants of the study for their contribution. We express special gratefulness to the professional translators for their priceless contribution and support.

\section{Authors' contributions}

K.S.: Project development, management and data collection, manuscript writing; E.S.: Management and data collection, manuscript writing; J.F.A.: management, manuscript editing; A.P.: Manuscript editing; K.G.N.: Project and protocol development, manuscript editing; F.M.W.: Manuscript editing, supervision.

\section{Funding}

The study has no external funding resource to report.

\section{Conflicts of Interest}

K.S. and E.S. have no conflicts of interest.

J.F.A., K.G.N., A.P., and F.M.W. are coauthors and copyright holders of the ACSS questionnaire.

J.F.A. declares a personal conflict of interest with Elsevier publishing company due to his disagreement with the policy of the company.

\section{Copyright issues}

The ACSS is copyrighted by the Certificate of Deposit of Intellectual Property in Fundamental Library of Academy of Sciences of the Republic of Uzbekistan, Tashkent (Registration number 2463; 26 August 2015) and the Certificate of the International Online Copyright Office, European Depository, Berlin, Germany (Nr. EU-01-000764; 21 October 2015). The rightsholders are Jakhongir Fatikhovich Alidjanov (Uzbekistan), Ozoda Takhirovna Alidjanova (Uzbekistan), Adrian Martin Erich Pilatz (Germany), Kurt Guenther Naber (Germany), Florian Martin Erich Wagenlehner (Germany).

Translations of the ACSS in other languages are available on the website: http://www.acss.world/downloads.html 


\section{References}

1 Colgan R, Williams M. Diagnosis and treatment of acute uncomplicated cystitis. Am Fam Physician 2011; 84: 771-776.

2 Nicolle LE. Epidemiology of urinary tract infections. Clinical Microbiology Newsletter 2002; 24: 135140.

3 Bent S, Nallamothu BK, Simel DL, Finn SD, Saint S. Does this woman have an acute uncomplicated urinary tract infection? JAMA 2002; 287: 2701-2710.

4 Anger J, Lee U, Ackerman AL, Chou R, Chughtai B, Clemens JQ et al. Recurrent Uncomplicated Urinary Tract Infections in Women: AUA/CUA/SUFU Guideline. J Urol 2019; 202: 282-289.

5 Gagyor I, Bleidorn J, Kochen MM, Schmiemann G, Wegscheider K, Hummers-Pradier E. Ibuprofen versus fosfomycin for uncomplicated urinary tract infection in women: randomised controlled trial. BMJ 2015; 351: h6544.

6 Kronenberg A, Butikofer L, Odutayo A, Muhlemann K, da Costa BR, Battaglia M et al. Symptomatic treatment of uncomplicated lower urinary tract infections in the ambulatory setting: randomised, double blind trial. BMJ 2017; 359: j4784.

7 Wagenlehner FM, Abramov-Sommariva D, Holler M, Steindl H, Naber KG. Non-Antibiotic Herbal Therapy (BNO 1045) versus Antibiotic Therapy (Fosfomycin Trometamol) for the Treatment of Acute Lower Uncomplicated Urinary Tract Infections in Women: A Double-Blind, Parallel-Group, Randomized, Multicentre, Non-Inferiority Phase III Trial. Urol Int 2018; 101: 327-336.

Alidjanov JF, Naber KG, Abdufattaev UA, Pilatz A, Wagenlehner FM. Reevaluation of the Acute Cystitis Symptom Score, a Self-Reporting Questionnaire. Part II. Patient-Reported Outcome Assessment. Antibiotics (Basel) 2018; 7.

Alidjanov JF, Naber KG, Abdufattaev UA, Pilatz A, Wagenlehner FME. Reevaluation of the Acute Cystitis Symptom Score, a Self-Reporting Questionnaire. Part I. Development, Diagnosis and Differential Diagnosis. Antibiotics (Basel) 2018; 7.

10 Acquadro C. Linguistic validation manual for patient-reported outcomes (PRO) instruments. Mapi Research Institute: Lyon, 2004.

11 Acquadro C, Conway K, Giroudet C, Mear I. Linguistic validation manual for health outcome assessments. Mapi Institute: Lyon, 2012.

12 Ervin S, Bower RT. Translation Problems in International Surveys. Public Opinion Quarterly 1952; 16: 595.

13 Alidjanov JF, Naber KG, Pilatz A, Wagenlehner FM. Validation of the American English Acute Cystitis Symptom Score. Antibiotics (Original paper) 2020; 9: 929.

14 Stamatiou K, Samara E. ACSS validation form for doctors. Google Forms, 2020.

15 contributors W. Greek language question. Wikipedia, The Free Encyclopedia, 2020.

16 Gkaragkouni O-M. The Sociolinguistic Phenomenon of Modern Greek Diglossia: the Outcome of Conflicts between $(\mathrm{H})$ igh and (L)ow Variety and the National Language Question in 19th 20th c. Greece: an Historico - Sociolinguistic Perspective. The ITB Journal 2009; 10.

Hello Yuqo. British VS American English: Why it's important to be specific. Yuqo: Web, 2018. 


\section{Legends of Figures and Tables}

Table 1. Age, level of education and preferred translated version of the first 15 females $(x)$ and the final group, including the first 15 females, comparing the pre-final Greek versions.

Table 2. Age, level of education and presence of history of acute cystitis of the 30 females participating commenting on the translated questions of the pre-final Greek versions.

Table 3. Age, level of education and presence of history of acute cystitis of the 30 females participating in the cognitive assessment process.

Figure 1. The final study version of the Greek ACSS questionnaire (Part A for the diagnosis and Part B for the follow-up) 